\title{
A Comprehensive Method for Assessing Meat Freshness Using Fusing Electronic Nose, Computer Vision, and Artificial Tactile Technologies
}

\author{
Xiaohui Weng, ${ }^{1,2,3}$ Xiangyu Luan, ${ }^{1}$ Cheng Kong, ${ }^{4}$ Zhiyong Chang $\mathbb{D}^{1,2}$ Yinwu Li, ${ }^{1,2}$ \\ Shujun Zhang $\mathbb{D}^{2,5}$ Salah Al-Majeed, ${ }^{5}$ and Yingkui Xiao ${ }^{1}$ \\ ${ }^{1}$ College of Biological and Agricultural Engineering, Jilin University, Changchun 130022, China \\ ${ }^{2}$ Key Laboratory of Bionic Engineering, Ministry of Education, Jilin University, Changchun 130022, China \\ ${ }^{3}$ School of Mechanical and Aerospace Engineering, Jilin University, Changchun 130022, China \\ ${ }^{4}$ College of Mathematics, Jilin University, Changchun 130012, China \\ ${ }^{5}$ School of Computing and Engineering, University of Gloucestershire, the Park, Cheltenham GL50 2RH, UK \\ Correspondence should be addressed to Zhiyong Chang; zychang@jlu.edu.cn and Shujun Zhang; szhang@glos.ac.uk
}

Received 7 May 2020; Revised 21 July 2020; Accepted 1 September 2020; Published 21 September 2020

Academic Editor: Alberto J. Palma

Copyright ( 2020 Xiaohui Weng et al. This is an open access article distributed under the Creative Commons Attribution License, which permits unrestricted use, distribution, and reproduction in any medium, provided the original work is properly cited.

The traditional methods cannot be used to meet the requirements of rapid and objective detection of meat freshness. Electronic nose (E-Nose), computer vision (CV), and artificial tactile (AT) sensory technologies can be used to mimic humans' compressive sensory functions of smell, look, and touch when making judgement of meat quality (freshness). Though individual E-Nose, CV, and AT sensory technologies have been used to detect the meat freshness, the detection results vary and are not reliable. In this paper, a new method has been proposed through the integration of E-Nose, CV, and AT sensory technologies for capturing comprehensive meat freshness parameters and the data fusion method for analysing the complicated data with different dimensions and units of six odour parameters of E-Nose, 9 colour parameters of CV, and 4 rubbery parameters of AT for effective meat freshness detection. The pork and chicken meats have been selected for a validation test. The total volatile base nitrogen (TVB-N) assays are used to define meat freshness as the standard criteria for validating the effectiveness of the proposed method. The principal component analysis (PCA) and support vector machine (SVM) are used as unsupervised and supervised pattern recognition methods to analyse the source data and the fusion data of the three instruments, respectively. The experimental and data analysis results show that compared to a single technology, the fusion of E-Nose, CV, and AT technologies significantly improves the detection performance of various freshness meat products. In addition, partial least squares (PLS) is used to construct TVB-N value prediction models, in which the fusion data is input. The root mean square error predictions (RMSEP) for the sample pork and chicken meats are 1.21 and 0.98 , respectively, in which the coefficient of determination $\left(R^{2}\right)$ is 0.91 and 0.94 . This means that the proposed method can be used to effectively detect meat freshness and the storage time (days).

\section{Introduction}

Meat contains a lot of easily digestible proteins, high-calorie fats, vitamins, and trace elements that are fundamental nutrients for human metabolism. So, meat is one of the most popular foods in the world [1]. From 2005, the average global meat consumption is about 110 grams per person per day [2]. Over the past 50 years, global meat production has almost quadrupled from 84 million tons in 1965 to a record of 335 million tons of meat in 2018 [3]. The quality of meat is directly related to the health of consumers [4]. With continuous increase of human beings' living standards, the quality and safety of food have been increasingly valued by consumers [5]. To meet the requirement of growing consumption and high-quality demanding, fresh meats have to be processed and transported in efficient and healthy ways. 
However, one significant problem is that the meat can be easily corrupted during processing and storage. In addition, some rogue traders sell metamorphic meats treated using chemical processes to make the metamorphic meats look fresh. The ordinary people cannot identify if the chemically treated meats are metamorphic or not. Consuming these kinds of meats imposes health risks.

Meat freshness is an important indicator of the quality and the safety of meat products, which attracts intense attention of meat producers, sellers, and consumers [6]. Traditional methods used for determining meat freshness include sensory evaluations, chemical measurements, and microbiological measurements [7]. Sensory evaluations use human senses to provide information such as colour, odour, rubbery state, and the overall quality of meat [8]. However, there are a number of shortcomings in sensory analysis, such as the high cost of the expert team, the judgment deviations due to fatigue and subjectivity, and the fact that sensory analysis cannot be used for online measurement [9]. In general, chemical methods include the measurements of meat $\mathrm{pH}$ [7], total volatile base nitrogen (TVB-N) [6], and 2,3,5-triphenyltetrazolium chloride (TTC) [10]. Chemical methods are objective and precise; however, they are normally used in laboratories, which are destructive and time-consuming [11]. Microbiological counting methods are also destructive and require a very long time for bacterial culture and cannot provide test results quickly and effectively [12]. Therefore, it is necessary to study new methods for efficient, cost-effective, and nondestructive meat freshness testing to ensure meat's quality and safety.

Previous studies have shown that some rapid and nondestructive analysis techniques have been proposed for meat freshness assessment, such as electronic nose (E-Nose) [1315], electronic tongue [16-18], computer vision (CV) [19$21]$, and spectroscopic technique [12, 22, 23]. Although these detection technologies have their own advantages, they can only be used to detect the certain aspects of meat freshness information; thus, they cannot be used to carry out a comprehensive assessment of meat freshness or spoilage [24]. For example, the E-Nose and the electronic tongue can only be used to detect the volatile odour and taste of the meat; the $\mathrm{CV}$ cannot be used to detect the chemical information of the meat, and the spectroscopic technique cannot be used to catch the spatial distribution information in the samples [25]. However, the freshness decline of meat products is a rather complicated process, which is related to the interaction of various factors such as microorganisms and enzymes [26]. The main components of meat, such as proteins and fats, are decomposed by enzymes and bacteria, and this process produces a large amount of volatile organic compounds. This process releases odours that are unpleasant to the senses. At the same time, the colour of the meat surface becomes dark, and the texture as the rubbery state decreases as well. So, the indicators of meat freshness should include odour, colour, and rubbery state. Therefore, it is important and necessary that the meat freshness detection methods should have the functions to comprehensively and accurately detect the meat freshness information from different perspectives (the above four indicators). Besides, the capability of detecting the length of storage of the meats should be another important feature of a meat freshness detection system.

Integrating a variety of nondestructive testing techniques can assess meat freshness from multiple perspectives. Nearinfrared spectroscopy, CV, and E-Nose technology were integrated into a method for detecting volatile base nitrogen in pork to assess pork freshness [27], and E-Nose and electronic tongue were also jointly employed in a new nondestructive detection method for fish freshness usage [28]. These studies mostly imitate human senses of smell, taste, and vision and use the fusion of artificial olfaction (E-Nose), artificial taste (electronic tongue), and artificial vision (CV) to evaluate meat freshness. But they do not involve mimicking human touch functions. If the structure of fresh meat is perfect, meat demonstrates good rubbery state and the proportion of the water holding capacity is high. With the decrease of its freshness, a series of biochemical reactions such as autolysis, bacterial growth, and reproduction in meat damage the integrity of the meat structure and hence reduce the rubbery state of muscle fibres. There is a clear relationship between the rubbery state of meat and the freshness. So, the rubbery state of meat should be used as an indicator for freshness evaluation. This is consistent with people's natural way for evaluating meat freshness by touching meats to sense its rubbery state.

A large amount of data is produced when using electronic measurement systems, so it is necessary to use statistical methods for analysing data for pattern recognition. Compared with a single analysis method, data fusion of complementary technology can provide more accurate sample knowledge and produce better inferences [29]. Electronic sensory measurements produce a large amount of data with various dimensions and units. The typical analysis methods are either nonsupervised or supervised algorithms. Principal component analysis (PCA) is a typical nonsupervised method used for pattern recognition. It creates new variables called principal components (PCs) from the linear combination of the original variables, and the purpose is to reduce the dimensionality of the data set while retaining most of the original variability in the data [30]. Support vector machine (SVM) is a supervised machine learning algorithm that assigns objects to specific classes through a training set. The basic principle of SVM is to create an optimal hyperplane that separates data belonging to different classes which has the highest possible confidence interval [31]. PCA and SVM, as unsupervised and supervised pattern recognition methods, have been effectively used in electronic sensory analysis of food quality, and good classification results have been achieved [32-34]. So, in this paper, both PCA and SVM will be used in designing and developing algorithms for data analysis.

In this paper, a new method is proposed through the integration of E-Nose, CV, and AT sensory technologies for capturing comprehensive meat freshness parameters and the data fusion method for analysing the complicated data with different dimensions and units of 6 odour parameters of ENose, 9 colour parameters of $\mathrm{CV}$, and 4 rubbery parameters of AT for effective meat freshness detection. The pork and 


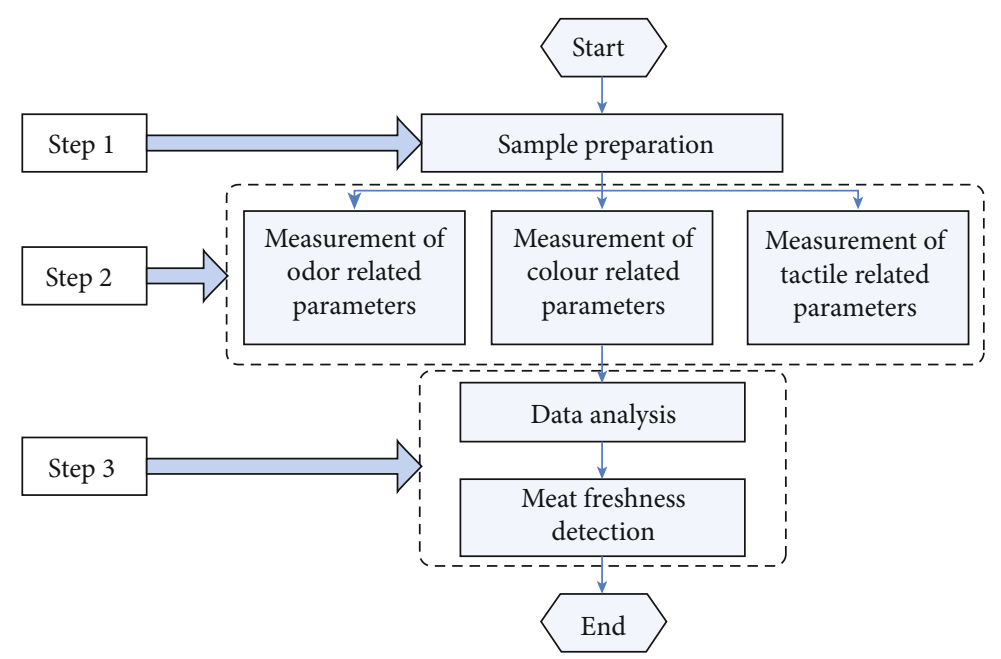

FIgURE 1: The proposed method for meat freshness detection.

chicken meats will be selected for validation test. The total volatile base nitrogen (TVB-N) assays are used to define meat freshness as the standard criteria for the validation of the effectiveness of the proposed method. It is expected that the proposed method can be used to effectively detect meat freshness and the storage time (days).

\section{Proposition of the New Methods for Detecting Meat Freshness}

2.1. Introduction. In this section, a new method was proposed for detecting meat freshness and the storage time in days using the fusion of E-Nose, CV, and AT to mimic human biosensing capabilities: olfactory, visual, and tactile. With the proposed method, all three aspects of meat's freshness, the flavour, colour, and texture as the rubbery state, are integrated. The procedure of the proposed method is shown in Figure 1 . There are three steps: Step $1-$ sample preparation, Step 2-measurement of freshness parameters, and Step 3-data analysis and meat freshness detection.

In Step 1, the number of the samples of the meat is firstly determined, and the samples should be processed following the standard meat experimental procedure. In Step 2, three kinds of measurements are designed for collecting the data related to odour, colour, and rubbery state and to store the data in a dedicated database. In Step 3, the data collected in Step 2 is analysed using data fusion analysis and pattern identification methods to detect the meat freshness.

2.2. Step 1: Sample Preparation. The preparation of the fresh meat samples should be carried out as soon as possible following the standard meat experimental procedure. The number of the samples of the meats is firstly determined. Then, the fresh meats should be cut on a clean bench with a sterile cutter into the desired sample thickness and weight for the test; the shape of the meat sample is cube-like with approximately $10 \pm 1 \mathrm{~mm}$ in side length and $30 \pm 1 \mathrm{~g}$ in weight. If the measurements cannot be carried out immediately, the prepared fresh meat samples should be kept in a refrigerator, at $8^{\circ} \mathrm{C}$.

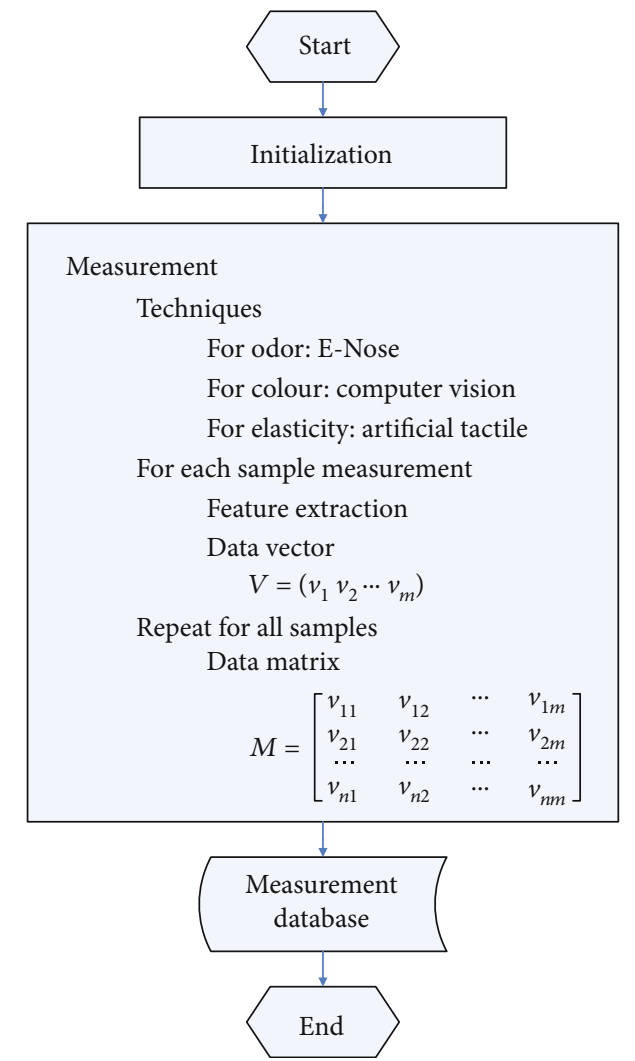

Figure 2: Procedures for measurement of related freshness parameters.

2.3. Step 2: Measurement of Freshness Parameters. In this step, three kinds of measurements are designed for collecting the data that is related to odour, colour, and rubbery and to store the data in a dedicated database, as show in Figure 2.

An E-Nose with gas sensor array is selected to collect odour-related meat freshness parameters. Then, the maximum response of each sensor is extracted as a feature variable for $i^{\text {th }}$ sample (1). Repeating measurement for all samples forms feature matrix (2) for odour. 


$$
\begin{aligned}
V_{E i} & =\left(\begin{array}{llll}
E_{i 1} & E_{i 2} & \cdots & E_{i p}
\end{array}\right), \\
M_{E} & =\left[\begin{array}{cccc}
E_{11} & E_{12} & \cdots & E_{1 p} \\
E_{21} & E_{22} & \cdots & E_{2 p} \\
\cdots & \cdots & \cdots & \cdots \\
E_{n 1} & E_{n 2} & \cdots & E_{n p}
\end{array}\right],
\end{aligned}
$$

where $p$ is the number of feature variables of the E-Nose and $n$ is the number of the samples.

To measure colour-related freshness parameters, a computer vision system is employed. A high-resolution camera is used to take images of meats; then, the colour information is extracted as a feature variable for $i^{\text {th }}$ sample using an image processing method (3). Repeating measurement for all samples obtains feature matrix (4) for colour.

$$
\begin{aligned}
V_{C i}= & \left(\begin{array}{llll}
C_{i 1} & C_{i 2} & \cdots & C_{i q}
\end{array}\right), \\
M_{C} & =\left[\begin{array}{cccc}
C_{11} & C_{12} & \cdots & C_{1 q} \\
C_{21} & C_{22} & \cdots & C_{2 q} \\
\cdots & \cdots & \cdots & \cdots \\
C_{n 1} & C_{n 2} & \cdots & C_{n q}
\end{array}\right],
\end{aligned}
$$

where $q$ is the number of feature variables of the computer vision system.

To measure elasticity-related freshness parameters, an artificial tactile system is used. Extracting the information related to the elasticity properties of the system yields the feature variables for $i^{\text {th }}$ sample (5). Repeating measurement for all samples produces feature matrix (6) for elasticity.

$$
\begin{aligned}
V_{A i} & =\left(\begin{array}{llll}
C_{i 1} & C_{i 2} & \cdots & C_{i d}
\end{array}\right), \\
M_{A} & =\left[\begin{array}{cccc}
A_{11} & A_{12} & \cdots & A_{1 d} \\
A_{21} & A_{22} & \cdots & A_{2 d} \\
\cdots & \cdots & \cdots & \cdots \\
A_{n 1} & A_{n 2} & \cdots & A_{n d}
\end{array}\right],
\end{aligned}
$$

where $q$ is the number of feature variables of the artificial tactile system.

2.4. Step 3: Data Analysis and Meat Freshness and Storage Time Detection. In Step 3, the collected data is analysed for detecting meat freshness with two outputs: (1) the duration of the storage in days and (2) the meat freshness, as show in Figure 3. There are three tasks: Task 1-data initial processing, Task 2-data fusion, and Task 3-recording time detection and meat freshness.

2.4.1. Task 1: Data Initial Processing. The data collected by the three measurement technologies have different dimensions. This dimension nonuniform issue should be firstly sorted out using a normalizing algorithm. The initialised data is then stored in separate data tables of a dedicated database.

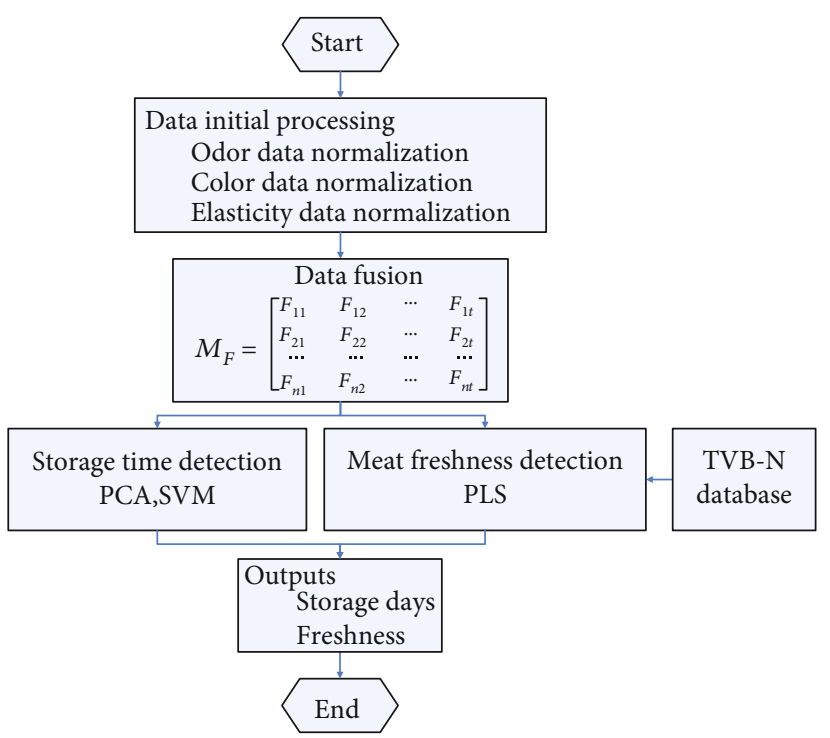

Figure 3: Procedures for data analysis and meat freshness detection.

2.4.2. Task 2: Data Fusion. Fuse the initialised data representing odour, colour, and rubbery state features of the meats using feature-level fusion methods.

2.4.3. Task 3: Recording Time Detection and Meat Freshness. Detect storage time using PCA and SVM algorithms, and detect meat freshness using the PLS algorithm with the TVB-N database.

It has been known that a series of complex biochemical reactions occurs during the process of spoilage of meat products, and the corrupted meat decomposes to produce basic nitrogenous substances such as ammonia and amines. These substances are volatile in alkaline environments and are therefore called volatile base nitrogen. It has been found that the changes of the meat freshness are related to the changes of the amount of volatile base nitrogen [35]. So TVB-N is an accurate and reliable method for evaluating the freshness of meats. Therefore, in this paper, TVB-N is used as the criterion for establishing the relationships between the meat freshness and the three kinds of parameters used in the proposed method.

\section{Materials and Methods}

3.1. Sample Preparation. In this paper, two typical kinds of meats with the different characteristics of odour, colour, and rubbery state have been selected for tests: pork and chicken. The meat samples were the fresh pork loins and fresh chicken breasts purchased in the local market in Changchun City, Jilin Province, China. The fresh meats were kept in the laboratory refrigerator immediately after purchase. The delivery time from the market to the laboratory was less than 1 hour. The pork loins and chicken breasts were cut on a clean bench with a sterile cutter into the small samples of approximately $30 \pm 1 \mathrm{~g}$ for the test. To minimise the effects of the meat freshness of the handling of the meat samples, there were no any other treatments before cutting and packaging. To increase the reliability of validation tests, 


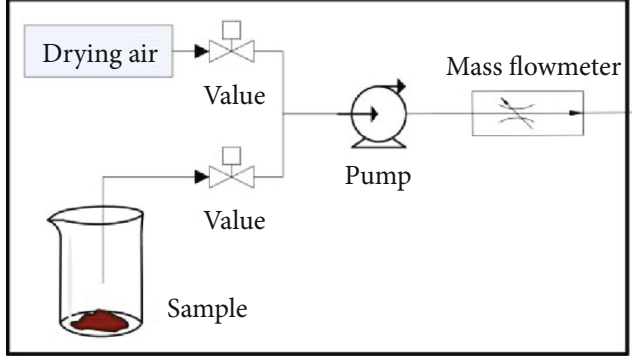

Gas supply device

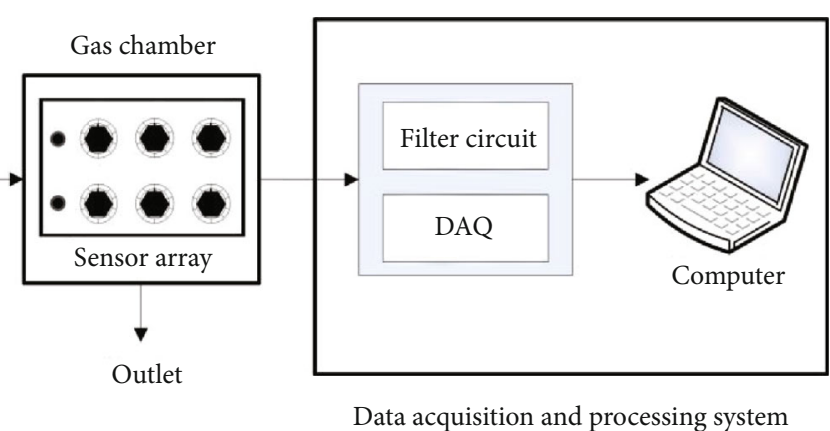

Data acquisition and processing system

Figure 4: The components of the E-Nose system.

twenty-eight samples of pork loins and chicken breasts were prepared, respectively. And then they were packed in commercial food preservation bags, sealed, and stored in a refrigerator at $8^{\circ} \mathrm{C}$.

In order to test the freshness of meat, the samples were stored for 7 days and experimented once a day. Eight experiments were carried out. The test of E-Nose, CV, and AT is nondestructive, but TVB-N is destructive. So, among 28 samples for both pork and chicken, 20 samples were used repeatedly for E-Nose, CV, and AT experiments. Remaining 8 samples were used for the TVB-N test. The TVB-N values of the two kinds of meats as the criteria for establishing the relationships between the meat freshness and the three kinds of parameters for obtaining generic rules for freshness detection.

\subsection{The Measurement of the Parameters of Meat Freshness}

3.2.1. Measurement of the Odour-Related Features Using the E-Nose System. The odour of meat was measured using an innovative E-Nose system developed by the authors, as shown in Figure 4. It consists of three units: gas supply unit, sensor array unit, data acquisition, and processing unit. The sensor array consists of six metal oxide semiconductor gas sensors capturing most required gases released from meats. The gas sensors used in this E-Nose are the TGS series manufactured by Figaro, Japan. The response characteristics of these sensors are shown in Table 1.

During the experiment, the E-Nose system was placed in a clean indoor air environment with an ambient temperature of $22 \pm 1^{\circ} \mathrm{C}$ and a humidity of $60 \pm 1 \%$. To obtain the stable and reliable measurement results, the sensors were preheated for one hour before the formal tests. The meat samples were placed in a $500 \mathrm{ml}$ beaker which was sealed with a plastic wrap and enhanced desorption of volatile compounds from the meat into the headspace for $15 \mathrm{~min}$ in the test environment. At the beginning of the measurement, the sample's headspace air was pumped into the sensor chamber at a flow rate of $300 \mathrm{ml} / \mathrm{min}$ using an air pump. The data acquisition frequency was $50 \mathrm{~Hz}$, and the acquisition time was $60 \mathrm{~s}$. After each measurement, dry air was blown into the E-Nose system for $15 \mathrm{~min}$ to completely clean it before the next experiment.

3.2.2. Measurement of the Colour-Related Features Using the Computer-Vision System. The surface images of the sample meats were captured in a $50 \times 50 \times 50 \mathrm{~cm}$ dark box with four
TABLE 1: Response characteristics of gas sensors.

\begin{tabular}{lccc}
\hline Name & Sensors & $\begin{array}{c}\text { Sensitivity } \\
\text { characteristics }\end{array}$ & $\begin{array}{c}\text { Typical detection } \\
\text { ranges }(\mathrm{ppm})\end{array}$ \\
\hline S1 & TGS2602 & $\begin{array}{c}\text { VOCs, ammonia, } \\
\text { hydrogen sulfide }\end{array}$ & $1-30$ \\
S2 & TGS2611 & Methane & $500-10,000$ \\
S3 & TGS2442 & Carbon monoxide & $30-1000$ \\
S4 & TGS4161 & Carbon dioxide & $350-10,000$ \\
S5 & TGS2620 & Alcohol & $500-5000$ \\
S6 & TGS2610 & Methane, butane & $500-10,000$ \\
\hline
\end{tabular}

${ }^{a}$ The response of sensors is nonspecific. They are also sensitive to other gases, which are not listed in this table.

standard bulbs (2U5W, Philips, Germany) placed in the four corners above the box. The samples were placed in the middle of the bottom in the box, and a digital camera (EOS 60D, Canon Inc., Japan) was fixed above the box and perpendicular to the sample. The resolution of the images is $5184 \times 3456$ pixels. Camera parameters were set as follows: shutter speed 1/80 seconds, manual operation mode, aperture AV F/10, ISO speed 320, and flash off. Before taking an image, a white card (white balance card, $21.59 \times 27.94 \mathrm{~cm}$, X-Rite) was used to customize the white balance for the calibration of the camera.

The captured images were preprocessed using MATLAB software for the late analysis.

3.2.3. Measurement of the Rubbery State-Related Feature Variables Using an AT System. To mimic the process of using a human hand to touch meats for "sensing" the rubbery state of meat, in this paper, the WDW-20J electronic universal testing machine (Shanghai Hualong Test Instruments Corporation, China) was selected, as shown in Figure 5. The upper part is the indenter, and the lower part is the tray. The indenter is connected to the pressure sensor. During the tests, the meat samples move with the tray vertically. In this study, 10 times of trial screening and comparative evaluation tests have been carried out. Based on the trial results, it was found that an indenter diameter of $8 \mathrm{~mm}$ and the vertical motion speed of the base tray of $100 \mathrm{~mm} / \mathrm{min}$ are the optimum combination of experimental parameters for the selected AT experiments. Besides, the tray was moved upwards and stopped at a vertical displacement of $5 \mathrm{~mm}$; 


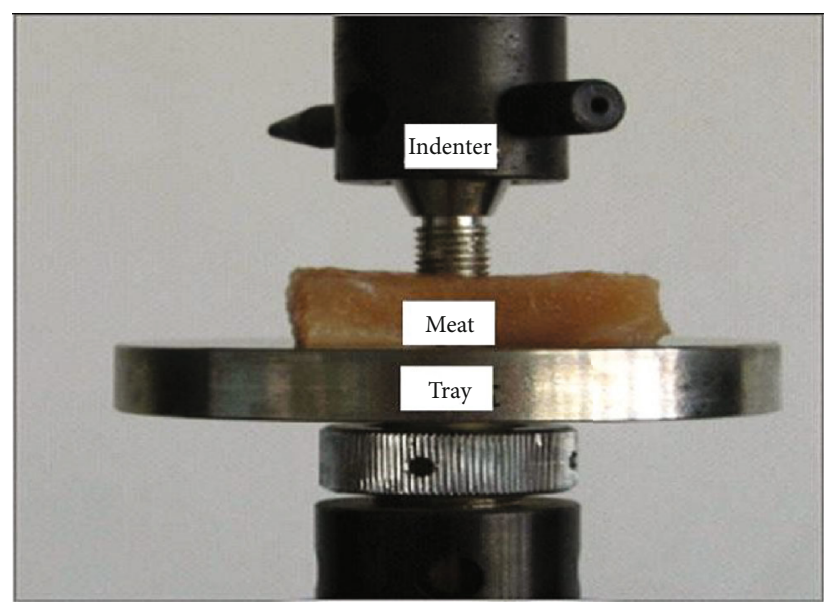

Figure 5: Schematic diagram of tactile test device.

then, the pressure was recorded before it returned to the starting point at the same speed.

Figure 6 shows a schematic diagram of the pressure characteristic curve of the meat samples. In the figure, $F$ is the pressure, $L_{1}$ is the maximum indentation depth when the meat is pressed, and $L_{2}$ is the indentation that cannot be recovered when the meat is in touch with the indenter.

3.2.4. The TVB-N Measurement. In this paper, the Chinese national standard method (GB/T 5009.44-2003) for TVB-N measurement has been used for obtaining TVB-N values [36], since this is a national standard method in China. So this method was adopted for the experiments in this paper.

In this method, there are 4 steps: Step $1-10 \mathrm{~g}$ meat is sampled, minced, and placed in a conical flask; Step 2-add $100 \mathrm{ml}$ purified water into sample, shake the mixture about $10 \mathrm{~s}$, and soak for $30 \mathrm{~min}$ in the test environment; Step 3 -load $5 \mathrm{ml}$ of the filtrate into Kjeldahl distillation apparatus, add $5 \mathrm{ml}$ of a $10 \mathrm{~g} / \mathrm{L}$ magnesium oxide solution, and let the filtrate distil for $5 \mathrm{~min}$; Step 4-absorb the distillate using $10 \mathrm{ml}$ of $20 \mathrm{~g} / \mathrm{L}$ boric acid absorption solution, and then titrate with $0.1 \mathrm{~mol} / \mathrm{L}$ hydrochloric acid solution. The TVB$\mathrm{N}$ value was captured and expressed in $\mathrm{mg} / 100 \mathrm{~g}$ [37]. Each experiment was repeated three times, and the average of the three TVB-N values was used as the measured values for comparative analysis and evaluation.

\subsection{Data Analysis}

3.3.1. Data Normalization Method. As discussed above, because the parameters for the three kinds of meat freshness parameters have different dimensions and unit magnitudes, it is necessary to normalize the data using a standardized transformation method, expressed in

$$
X_{i k}=\frac{X_{i k}-\overline{X_{k}}}{S_{k}} \quad(i=1,2, \cdots, n ; k=1,2, \cdots, P),
$$

where $\overline{X_{k}}=(1 / n) \sum_{i=1}^{n} X_{i k}$ and $S_{k}=\sqrt{(1 /(n-1)) \sum_{i=1}^{n}\left(X_{i k}-\overline{X_{k}}\right)}$.

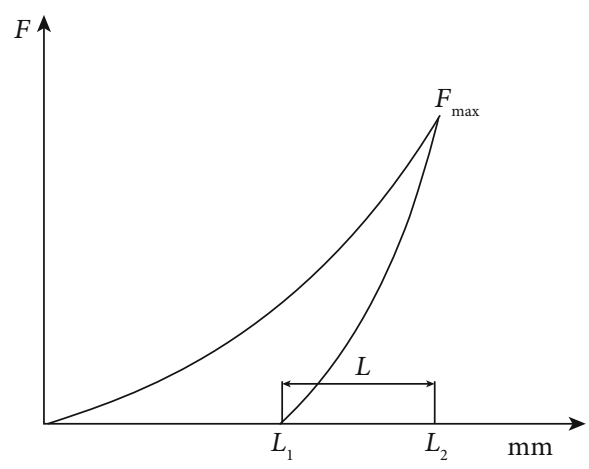

FIgURe 6: Pressure characteristic curve of meat sample.

3.3.2. Data Fusion Method. Data fusion is the process of integrating multiple data sources to produce more consistent, accurate, and useful information than that provided by any individual data source. In general, data fusion methods can be divided into three levels: data-level fusion (low level), feature-level fusion (medium level), and decision-level fusion (high level). Data-level fusion directly fuses the raw data. The data of all sources is simply organised into a matrix in order. Feature-level fusion is to extract feature values for all data sources and then associate the feature values to form an array for multivariate classification and regression. Decision-level fusion calculates independent classifications or models from each data source and combines the results of each model to make comprehensive decisions [38]. Data-level fusion has the advantages of retaining majority overall information and higher accuracy. However, due to the fact that the amount of raw data of all sources can be very large, computation is heavy and it takes long time for reaching decisions so it is not feasible to use data-level fusion for real-time analysis. Decision-level fusion has the advantages of processing a relatively small amount of data and good fault tolerance, but a large amount of data has to be preprocessed, resulting in considerable information loss [9]. Feature-level fusion has the advantages of processing the moderate amount of data, modest degree of computational complexity, and reliable fusion performance. Therefore, in this paper, a feature-level fusion method is employed to establish a multisensor information fusion recognition model.

\section{Data Analysis and Meat Freshness Detection}

4.1. TVB-N Analysis. The TVB-N content is an important indicator for evaluating the freshness of meat [39]. Figure 7 shows the experimental results of the changes of TVB-N amounts with the storage time of both the pork and chicken meat samples. As expected, the TVB-N amounts of both pork and chicken samples increased with storage time. This is due to the fact that the number of spoilage organisms increases as storage time increases, and the production of amines and other basic nitrogen compounds increases so that the TVB$\mathrm{N}$ production rises. According to the livestock and poultry meat freshness standard, if the TVB-N value is less than $15 \mathrm{mg} / 100 \mathrm{~g}$, the meat is fresh; if the TVB-N value is between 15 and $25 \mathrm{mg} / 100 \mathrm{~g}$, the meat is subfresh; if the TVB-N value 


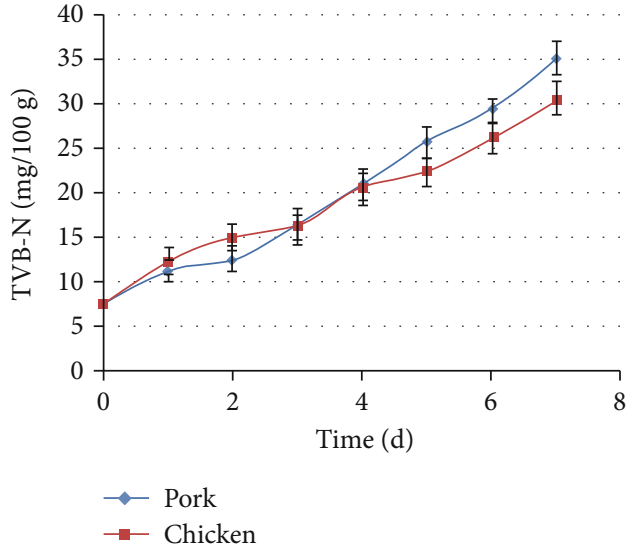

Figure 7: The experimental results of the changes of TVB-N contents of the samples with storage time.

is more than $25 \mathrm{mg} / 100 \mathrm{~g}$, the meat is completely rotten [37], as shown in Table 2. From Figure 7, it can be seen that (1) the pork and chicken samples were fresh in the first 2 days, (2) the quality declined from the third day, and (3) pork samples were fresh on the 3rd and 4th days and metamorphic on the 5 th day, while chicken samples were fresh on the 3rd to 5th days and metamorphic on the sixth day.

4.2. E-Nose Analysis for Storage Time Detection. Figure 8 shows the responses of six MOS sensors for pork (Figure 8(a)) and chicken (Figure 8(b)) were stored for 2 days. It can be seen that the across-sensitivity response of sensors to target odours is indicated by the wide variability of the outputs of sensors. In addition, the sensors have different responses to pork and chicken, which indicates that the composition and contents of volatile gases in the two kinds of meats are different. However, the variation trend of the signals of different sensors in meats is similar, gradually increasing from $5 \mathrm{~s}$ to $20 \mathrm{~s}$ and then becoming steady after $20 \mathrm{~s}$. This is because the headspace gas gradually contacts the sensor from $5 \mathrm{~s}$. Sensor S5 (TGS2620) is the most sensitive to the headspace gas of both pork and chicken, yet sensor S2 (TGS2611) reacts minimally. This indicates that there are more alcohols and less alkane contents in the volatile components of meats.

The maximum value of each sensor is selected as the characteristic variable for PCA analysis. Figure 9 shows the results of PCA analysis of pork (Figure 9(a)) and chicken (Figure 9(b)) at different storage times. For the pork, PC1 and PC2 cumulatively contribute $90.30 \%$ of the variance in data while this value for the chicken is $91.37 \%$, which shows that the first two principal components can provide the sufficient information to explain the difference in the smell of the pork and chicken emitted during different storage time. From the projection of the two-dimensional plane composed of $\mathrm{PC} 1$ and $\mathrm{PC} 2$, it can be seen that fresh meat, subfresh meat, and rotten meat are obviously distributed in different regions. For the pork, fresh meat (0-2 days) is in the upper part of the figure, sub-fresh meat (3-4 days) is in the right side of the figure, and the rotten meat (5-7 days) is in the lower left part of the figure. For the chicken, fresh meat (0-2 days)
TABLE 2: Relationship between meat freshness and TVB-N [37].

\begin{tabular}{lc}
\hline Freshness & TVB-N $(\mathrm{mg} / 100 \mathrm{~g})$ \\
\hline Fresh & Less than 15 \\
Subfresh & Between 15 and 25 \\
Rotten & More than 25 \\
\hline
\end{tabular}

is in the lower left part of the figure, subfresh meat (3-5 days) is in the upper part of the figure, and rotten meat (6-7 days) is in the lower right part of the figure. At the same time, it is possible to distinguish the meat samples from different storage days. So based on the PCA analysis, our electronic nose can be used to classify the smell of meat at different storage times perfectly, which is consistent with previous research by some scholars $[40,41]$.

4.3. CV Analysis for Storage Time Detection. In order to eliminate the interference of the shadow around the image and the edge information of the meat, the $200 \times 200$ pixel area of the middle of the image was selected for analysis. According to a previous literature, most studies used the extracted image RGB (red, green, blue) colour models, HSI (hue, saturation, intensity) and $\mathrm{L} * \mathrm{a} * \mathrm{~b} *$ colour space information as sources of data for CV analysis $[42,43]$, and it achieves very good classification results. RGB is a hardware-oriented colour model. HIS is a colour model developed to adapt to human eyes' perception and description of colour. $\mathrm{L} * \mathrm{a} * \mathrm{~b}$ $*$ is independent with hardware, so it makes up for the shortage that RGB mode depends on the colour characteristics of equipment. The 3 colour models offer more information from different colour patterns. Therefore, in this paper, the RGB mean $\left({ }^{-} \mathrm{R},{ }^{-} \mathrm{G},{ }^{-} \mathrm{B}\right)$, HIS mean $\left({ }^{-} \mathrm{H},{ }^{-} \mathrm{S},{ }^{-} \mathrm{I}\right)$, and $\mathrm{L} * \mathrm{a} *$ $\mathrm{b} *$ mean $\left({ }^{-} \mathrm{L} *,{ }^{-} \mathrm{a} *,{ }^{-} \mathrm{b} *\right)$ of the image were extracted as the characteristic variables for the statistical analysis of the samples.

Figure 10 shows the PCA analysis of CV data for pork (Figure 10(a)) and chicken (Figure 10(b)) at different storage times. The contribution variances of cumulative data for pork and chicken by PC1 and PC2 are $92.03 \%$ and $92.37 \%$, respectively. From the projection of the two-dimensional plane composed of $\mathrm{PC} 1$ and PC2, it can be seen that fresh meat, subfresh meat, and rotten meat are clearly distributed in different regions. For the pork, the fresh meat (0-2 days) is in the upper part of the figure, the subfresh meat (3-4 days) is in the right side of the figure, and the rotten meat (5-7 days) is in the lower left part of the figure. For the chicken, the fresh meat (0-2 days) is in the upper left corner of the figure, the subfresh meat (3-5 days) is in the upper middle of the figure, and the rotten meat (6-7 days) is in the lower right part of the figure. At the same time, it is possible to distinguish the meat samples from different storage days. So, based on PCA analysis, it is believed that the CV is another perfect technology to distinguish meat odours at different storage times.

4.4. AT Analysis for Storage Time Detection. In AT, there are four parameters: the maximum pressure $\left(F_{\max }\right)$, the work done by the touch process $\left(W_{1}\right)$, the work done by the 


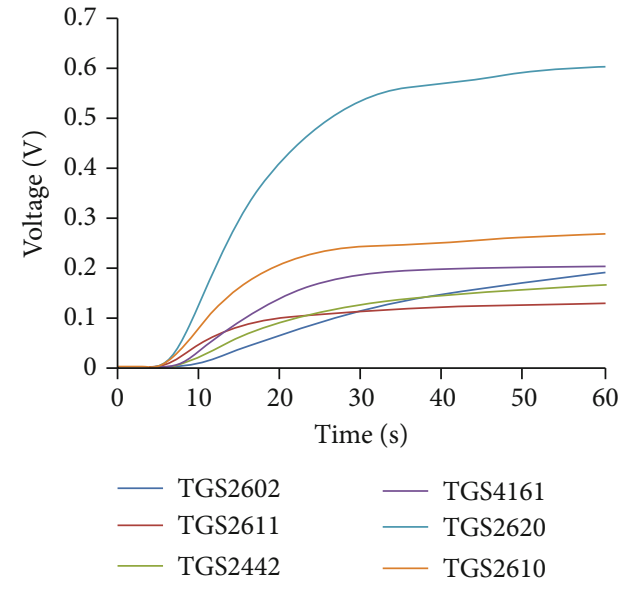

(a)

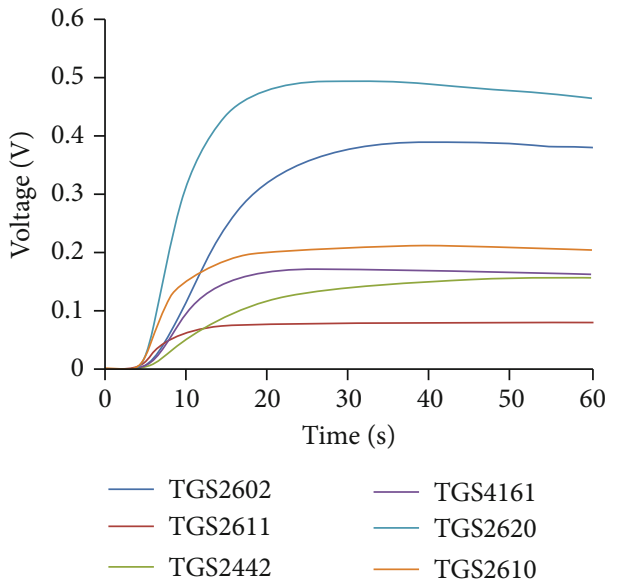

(b)

FIGURE 8: The responses of sensors for pork (a) and chicken (b) stored for 2 days.

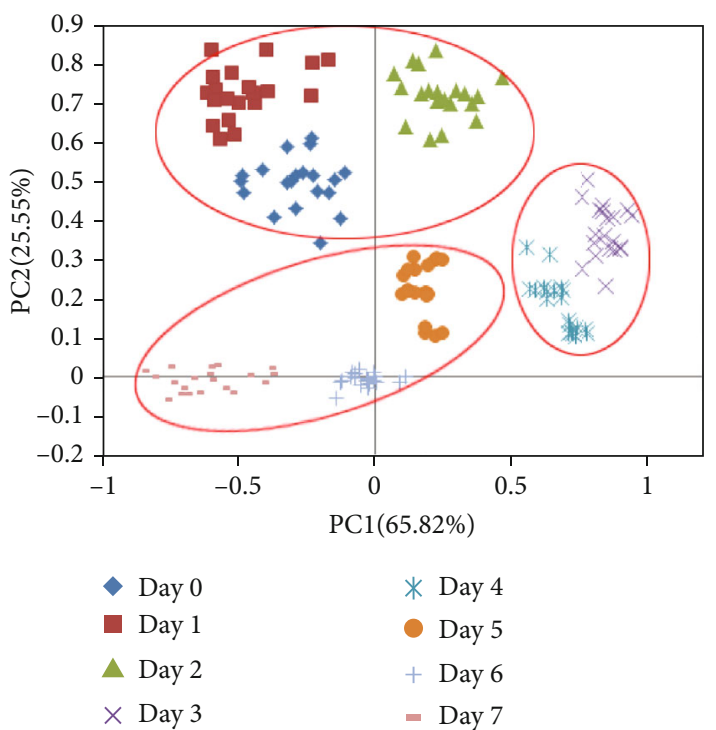

(a)

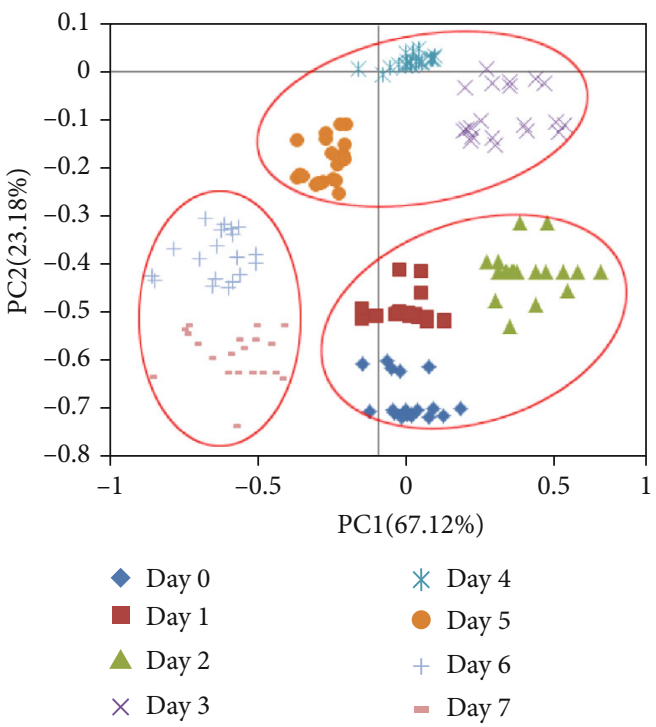

(b)

Figure 9: PCA plots of E-Nose analysis for pork (a) and chicken (b) at different storage times.

rebound process $\left(W_{2}\right)$, and the rebound amount $(L)$. They are the typical characteristic variables of the artificial haptic system. They can be expressed as

$$
\begin{gathered}
W_{1}=\int_{0}^{L_{2}} F d x, \\
W_{2}=\int_{L_{1}}^{L_{2}} F d x, \\
L=L_{2}-L_{1} .
\end{gathered}
$$

Figure 11 shows the PCA analysis results of AT data for pork (Figure 11(a)) and chicken (Figure 11(b)) at different storage times. The cumulative contribution variances of the first two principal components of pork and chicken are $82.01 \%$ and $87.73 \%$, respectively, so these first two PCs repre- sent most of the information. However, it can be seen that the PCA projection cannot be used to distinguish meat samples with different storage times. This is because the changes in meat freshness are a complex biochemical change, in which the meat structure is gradually destroyed; the rubbery state of meat has large nonlinear deformation and viscoelastic material properties [44]. PCA as a linear pattern recognition tool cannot use to solve this kind of classification problems. So it can be concluded that it is not feasible to use the data from a single kind of sensors for the meat freshness detection. Therefore, in this paper, the multisensor data fusion technology will be used for further analysis to achieve the reliable meat freshness detection.

4.5. Data Fusion Analysis for Storage Time Detection. The multisensor data fusion technology can be used to effectively integrate the outputs of multiple instrument source data for 


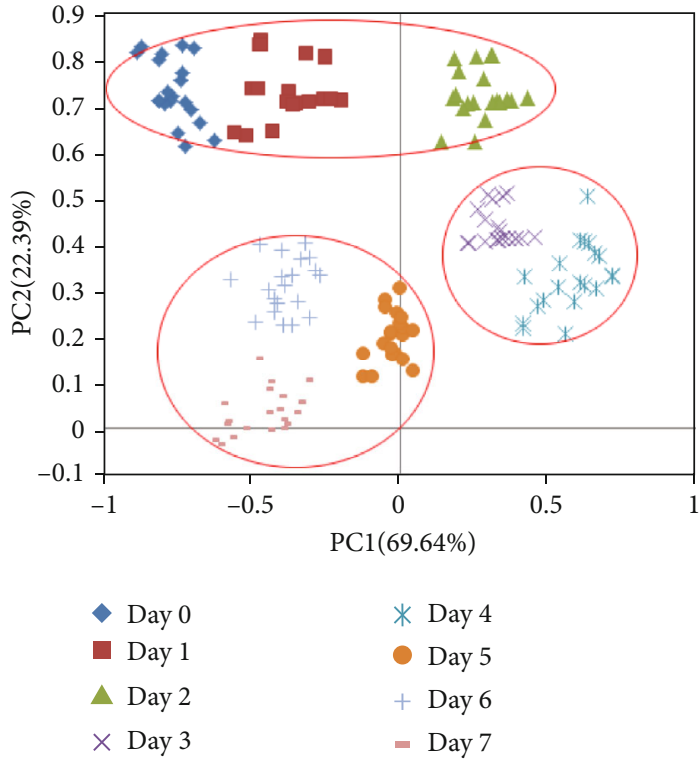

(a)

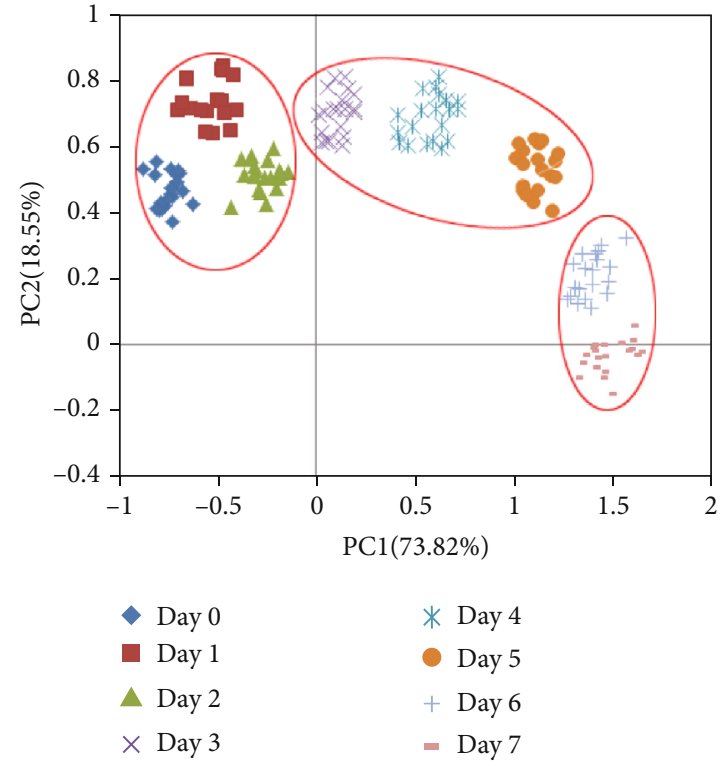

(b)

FIgURe 10: PCA plots of CV analysis for pork (a) and chicken (b) at different storage times.

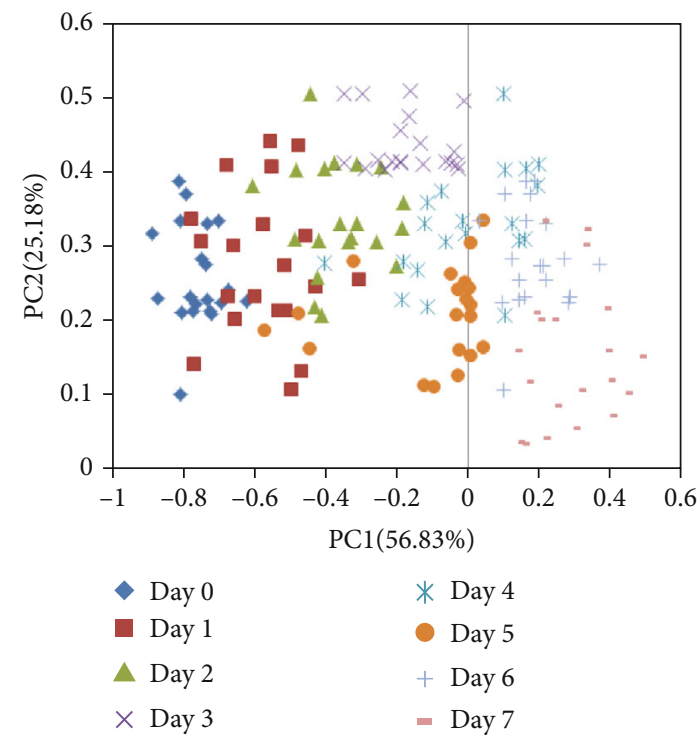

(a)

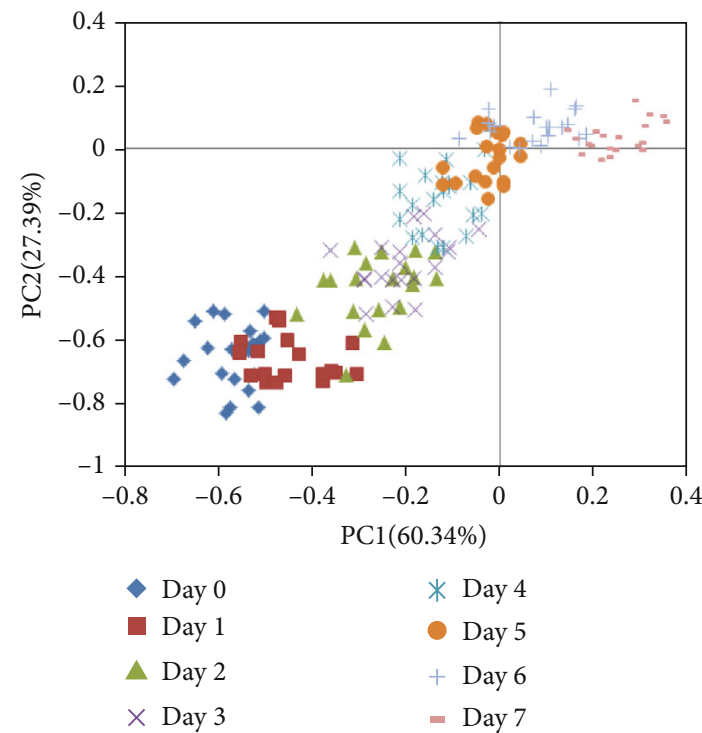

(b)

Figure 11: PCA plots of artificial visual analysis for pork (a) and chicken (b) at different storage times.

reliable food quality assessment and certification [29]. The midlevel fusion strategy involves the fusion of the original data matrix feature variables. In this study, 6 E-Nose feature variables, $9 \mathrm{CV}$ feature variables, and $4 \mathrm{AT}$ feature variables were used to form a new characteristic matrix for PCA analysis. Figure 12 shows the results of PCA analysis of fusion data for the pork (Figure 12(a)) and the chicken (Figure 12(b)) at different storage times. The first two main components represent most of the information (93.47\% for the pork and $95.40 \%$ for the chicken). In addition, the PCA projection plots are clearly divided into three zones repre- senting fresh meat, subfresh meat, and rotten meat. For the pork, the fresh meat (0-2 days) is in the lower right of the figure, the subfresh meat (3-4 days) is in the upper right of the figure, and the rotten meat (5-7 days) is in the left side of the figure. For the chicken, the fresh meat (0-2 days) is in the right side of the figure, the subfresh meat (3-5 days) is in the middle of the figure, and the rotten meat (6-7 days) is in the left side of the figure. At the same time, it is possible to distinguish meat samples from different storage days. Compared with E-nose and CV, the PCA results of the data fusion of the three detection techniques show that the 


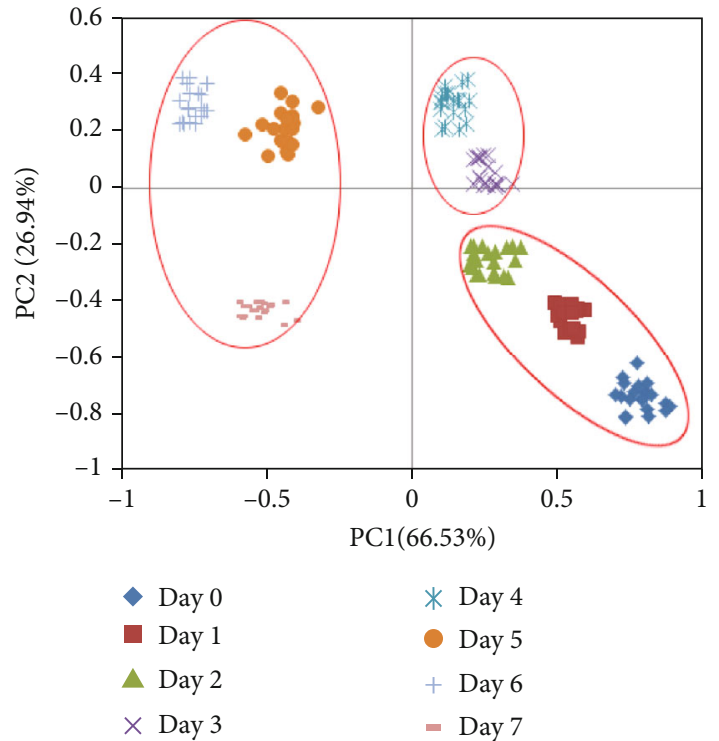

(a)

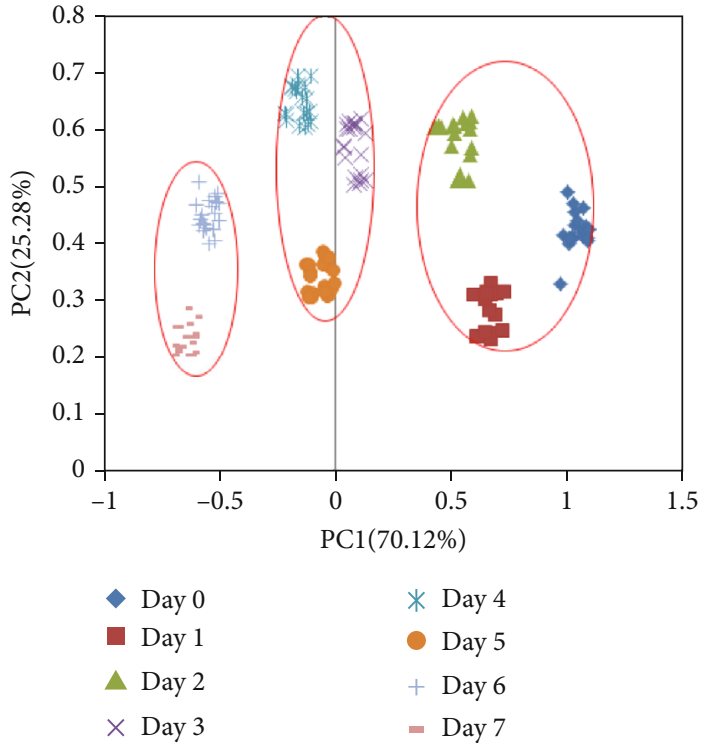

(b)

Figure 12: PCA plots of fusion information of pork (a) and chicken (b) at different storage times.

TABle 3: Comparison of identification rates of the pork and the chicken under different data variables.

\begin{tabular}{lcccc}
\hline Models & Pork & & Chicken & Calibration (\%) \\
E-Nose & Calibration (\%) & Prediction (\%) & 91.1 & 89.3 \\
CV & 92.7 & 88.2 & 87.1 & 86.8 \\
AT & 88.3 & 85.5 & 72.3 & 70.5 \\
E-Nose and CV & 72.3 & 69.5 & 92.8 & 90.1 \\
E-Nose and AT & 91.9 & 90.3 & 88.0 & 80.3 \\
CV and AT & 87.2 & 85.3 & 81.9 & 79.3 \\
E-NOSE, CV, and AT & 81.7 & 80.5 & 96.4 & 93.8 \\
\hline
\end{tabular}

projection is more aggregated and this data fusion analysis method can be used to clearly distinguish the meat samples with different freshness levels and storage times.

To further verify the effectiveness of this fusion method, this study continues to use the supervision mode identification method (SVM) for the sample classification, using the single detection method and the combination method for data modelling, respectively. The model input is consistent with the PCA model input above and is a feature variable extracted by each detection method. The radial basis function is used as the kernel function of the SVM. The penalty parameter $C$ and the kernel function parameter $\gamma$ are optimized using the grid search algorithm. The optimal values have been obtained for parameters $C=0.48539$ and $\gamma=5$. The classification results are shown in Table 3.

Table 4 shows the values of the quality metrics of the classification models under different data variables. The sensibility and specificity were 0.91 and 0.71 for pork and 0.92 and 0.70 for chicken.

From Tables 3 and 4 , it can be seen that

(1) if the single detection method is used for SVM identification, the E-Nose has the most important effect, and the AT recognition rate is the lowest, which is consistent with the results of PCA analysis

(2) if the two detection methods for data fusion are used for SVM recognition, the effect of the fusing E-Nose and CV is the most prominent, and the effect of fusing $\mathrm{CV}$ and $\mathrm{AT}$ is the lowest

(3) the effect of fusing three methods is better than all other methods

(4) the data fusion effect of multiple detection methods represents the rich information of the meat freshness, which is in line with the research results of Huang et al. [27]

Besides, the above analysis shows that the volatile odour emitted during the process of the meat spoilage is most related to its freshness, while the rubbery state and freshness have the smallest correlation. The data fusion of the two detection methods also illustrates this phenomenon.

4.6. Fusion Data Analysis for Meat Freshness Detection. The above analyses have shown that the data fusion of E-Nose, 
TABLE 4: Values of the quality metrics of the pork and the chicken under different data variables.

\begin{tabular}{lcccc}
\hline Models & Sensibility & Pork & Specificity & Sensibility \\
\hline E-Nose & 0.89 & 0.61 & 0.88 & Specificity \\
CV & 0.87 & 0.61 & 0.82 & 0.67 \\
AT & 0.65 & 0.45 & 0.66 & 0.61 \\
E-Nose and CV & 0.88 & 0.65 & 0.85 & 0.40 \\
E-Nose and AT & 0.82 & 0.61 & 0.84 & 0.77 \\
CV and AT & 0.75 & 0.57 & 0.92 & 0.58 \\
E-NOSE, CV, and AT & 0.91 & 0.71 & 0.70 \\
\hline
\end{tabular}

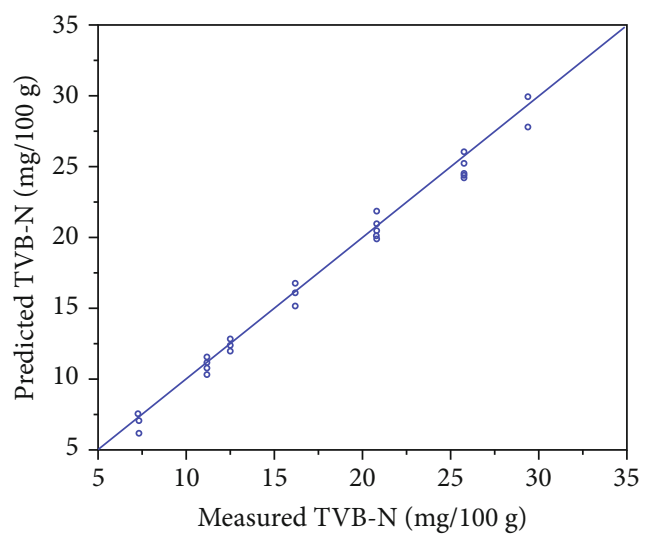

(a)

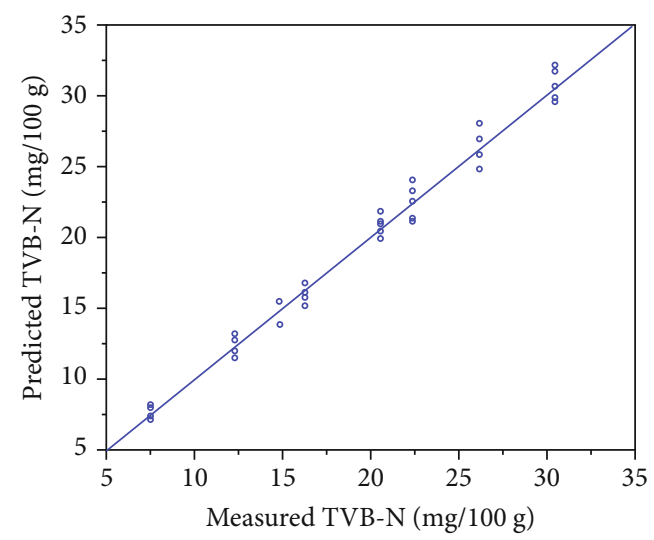

(b)

FIGURE 13: PLS model prediction results of the pork (a) and chicken (b).

$\mathrm{CV}$, and AT technologies can effectively distinguish meat samples of different storage times and hence can be used for detecting the storage days of the meats. In this section, the data fusion method is used to predict meat freshness. Since TVB-N is the widely accepted standard for assessing the freshness of meat, it is feasible to use TVB-N to predict the freshness of meats. In this paper, the PLS regression model was firstly established and the fusion data of the three methods was used as the model inputs and the outputs of the TVB-N values. Figure 13 shows PLS model prediction results of the pork and chicken. The $x$-axis is the measurement, and the $y$-axis is the prediction. The ideal prediction is that all points will be diagonally aligned along the graph and the predicted and measured values are the same. As can be seen from the figure, all the points are closely located around the diagonal line, which indicates that the prediction results are satisfactory. In addition, the root mean square error (RMSEP) of the pork and chicken predictions is 1.21 and 0.98 , respectively, and the coefficient of the determination $\left(R^{2}\right)$ is 0.91 and 0.94 , respectively, which also demonstrates the high correlation between fusion data and TVB-N analysis. These results confirm that the fusion of the three detection techniques can be used to reliably predict the meat freshness.

\section{Discussions}

Based on the test and data analysis results in Section 4, the following findings can be summarised.
E-Nose and CV technologies can be used to detect the storage time of the meats while AT cannot. The freshness detection accuracy of E-Nose is the highest, followed by CV and then by AT, which means that the most variable parameter of meat freshness is odour and the least variable parameter is rubbery. Though individual E-Nose, CV, and AT sensory technologies have been used to detect the meat freshness, the detection results vary and are not reliable. So these three technologies should be applied in the integrated way for the effective and reliable detection of the meat freshness. Data fusion analysis methods can be used to analyse the data with different dimensions and units, from the various sensors used in the system. The proposed method can be used to detect both the meat storage time (days) and meat freshness.

Different fusion methods have been proposed and reported to have relatively satisfactory performance for meat freshness detection, such as E-Nose, CV, near infrared spectroscopy fusion [27], and E-Nose and electronic tongue fusion [28]. Comparing these methods with the method proposed in this paper, near infrared spectroscopy equipment is complex, sensitive to environmental interference, of high price, and not feasible to commercial applications. An electronic tongue sensor is easily affected by environmental conditions, i.e., temperature and humidity, which may cause sensor drift. The fusion of E-Nose, CV, and AT does not have those shortcomings and is easy to develop into a commercial meat freshness detection tool. 
Though the above findings are meaningful for the future design and development of the systems for the on-line and real-time meat freshness detection and storage time in days, more experiments should be carried out on the large meat samples to obtain the sufficient data for optimising the system design and increasing data analysis accuracy.

\section{Conclusion}

Meat freshness detection is important to meat safety. The traditional methods cannot be used to meet the requirements of rapid and objective detection of meat freshness. E-Nose, CV, and AT sensory technologies which can be used mimic humans' compressive sensory functions of smell, look, and touch when making judgement of meat quality (freshness).

Though individual E-Nose, CV, and AT sensory technologies have been used to detect the meat freshness, the detection results vary and are not reliable. So these three technologies should be applied in the integrated way for the effective and reliable detection of the meat freshness.

Data fusion analysis methods can be used to analyse the data with different dimensions and units, from the various sensors used in the system.

A new method has been proposed through the integration of E-Nose, CV, and AT sensory technologies and data fusion analysis algorithms for effective meat freshness detection.

The pork and chicken meats have been selected for the validation test. The unsupervised (PCA) and supervised (SVM) pattern recognition and data fusion methods have been applied for analysing the complicated data with different dimensions and units of six odour parameters of E-Nose, 9 colour parameters of $\mathrm{CV}$, and 4 rubbery parameter of AT. The experimental and data analysis results show that the proposed method can be used to detect both the meat storage time (days) and meat freshness.

Though the findings in this paper are meaningful for the future design and development of the systems for the on-line and real-time meat freshness detection, more experiments should be carried out on the large meat samples to obtain the sufficient data for optimising the system design and increasing data analysis accuracy.

\section{Data Availability}

The data used to support the findings of this study are available from the corresponding author upon request.

\section{Conflicts of Interest}

The authors declare that they have no known competing financial interests or personal relationships that could have appeared to influence the work reported in this paper.

\section{Acknowledgments}

This work was supported by the National Natural Science Foundation of China (No. 51875245), the Special Project of Industrial Technology Research and Development of Jilin
Province (No. 2017C051-3), the Science and Technology Development Plan Project of Jilin Province (Nos. 20190201019JC and 20190303012SF), the Changchun Science and Technology Project (No. 18DY007), and the "13th Five-Year Plan" Scientific Research Foundation of the Education Department of Jilin Province (No. JJKH20190190KJ).

\section{References}

[1] W. Wojnowski, T. Majchrzak, T. Dymerski, J. Gębicki, and J. Namieśnik, "Electronic noses: powerful tools in meat quality assessment," Meat Science, vol. 131, pp. 119-131, 2017.

[2] S. De Smet and E. Vossen, "Meat: the balance between nutrition and health. A review," Meat Science, vol. 120, pp. 145156, 2016.

[3] World meat production to reach record high in, Food and Agriculture Organization of the United Nations, 2018, March 2020, https://www.globalagriculture.org/whats-new/news/en/ 33468.html.

[4] Y. Xiao, J. Jiaojiao, H. Guohua et al., "Determination of the freshness of beef strip loins ( $M$. longissimus lumborum) using electronic nose," Food Analytical Methods, vol. 7, no. 8, pp. 1612-1618, 2014.

[5] Y. N. Chen, D. W. Sun, J. H. Cheng, and W. H. Gao, "Recent advances for rapid identification of chemical information of muscle foods by hyperspectral imaging analysis," Food Engineering Reviews, vol. 8, no. 3, pp. 336-350, 2016.

[6] L. Qiao, X. Tang, and J. Dong, "A feasibility quantification study of total volatile basic nitrogen (TVB-N) content in duck meat for freshness evaluation," Food Chemistry, vol. 237, no. 117, pp. 1179-1185, 2017.

[7] Q. Chen, Z. Hui, J. Zhao, and Q. Ouyang, "Evaluation of chicken freshness using a low-cost colorimetric sensor array with AdaBoost-OLDA classification algorithm," LWT - Food Science and Technology, vol. 57, no. 2, pp. 502-507, 2014.

[8] O. S. Papadopoulou, E. Z. Panagou, F. R. Mohareb, and G. J. E. Nychas, "Sensory and microbiological quality assessment of beef fillets using a portable electronic nose in tandem with support vector machine analysis," Food Research International, vol. 50, no. 1, pp. 241-249, 2013.

[9] S. Kiani, S. Minaei, and M. Ghasemi-Varnamkhasti, "Fusion of artificial senses as a robust approach to food quality assessment," Journal of Food Engineering, vol. 171, pp. 230-239, 2016.

[10] B. Kuswandi and A. Nurfawaidi, "On-package dual sensors label based on $\mathrm{pH}$ indicators for real-time monitoring of beef freshness," Food Control, vol. 82, pp. 91-100, 2017.

[11] K. I. Sallam and K. Samejima, "Microbiological and chemical quality of ground beef treated with sodium lactate and sodium chloride during refrigerated storage," $L W T$ - Food Science and Technology, vol. 37, no. 8, pp. 865-871, 2004.

[12] H. Vasconcelos, C. Saraiva, and J. M. M. M. de Almeida, "Evaluation of the spoilage of raw chicken breast fillets using Fourier transform infrared spectroscopy in tandem with chemometrics," Food and Bioprocess Technology, vol. 7, no. 8, pp. 2330-2341, 2014.

[13] F. Mohareb, O. Papadopoulou, E. Panagou, G. J. Nychas, and C. Bessant, "Ensemble-based support vector machine classifiers as an efficient tool for quality assessment of beef fillets from electronic nose data," Analytical Methods, vol. 8, no. 18, pp. 3711-3721, 2016. 
[14] X. Tang, X. Sun, V. C. H. Wu et al., "Predicting shelf-life of chilled pork sold in China," Food Control, vol. 32, no. 1, pp. 334-340, 2013.

[15] V. Y. Musatov, V. V. Sysoev, M. Sommer, and I. Kiselev, "Assessment of meat freshness with metal oxide sensor microarray electronic nose: a practical approach," Sensors and Actuators B: Chemical, vol. 144, no. 1, pp. 99-103, 2010.

[16] L. Gil, J. M. Barat, D. Baigts et al., "Monitoring of physicalchemical and microbiological changes in fresh pork meat under cold storage by means of a potentiometric electronic tongue," Food Chemistry, vol. 126, no. 3, pp. 1261-1268, 2011.

[17] I. M. Apetrei and C. Apetrei, "Application of voltammetric etongue for the detection of ammonia and putrescine in beef products," Sensors and Actuators, B: Chemical, vol. 234, pp. 371-379, 2016.

[18] I. M. Apetrei, M. L. Rodriguez-Mendez, C. Apetrei, and J. A. de Saja, "Fish freshness monitoring using an E-tongue based on polypyrrole modified screen-printed electrodes," IEEE Sensors Journal, vol. 13, no. 7, pp. 2548-2554, 2013.

[19] M. Dowlati, M. de la Guardia, M. Dowlati, and S. S. Mohtasebi, "Application of machine-vision techniques to fish-quality assessment," TrAC Trends in Analytical Chemistry, vol. 40, pp. 168-179, 2012.

[20] M. Dowlati, S. S. Mohtasebi, M. Omid, S. H. Razavi, M. Jamzad, and M. de la Guardia, "Freshness assessment of gilthead sea bream (Sparus aurata) by machine vision based on gill and eye color changes," Journal of Food Engineering, vol. 119, no. 2, pp. 277-287, 2013.

[21] J. Lu, J. Tan, P. Shatadal, and D. E. Gerrard, "Evaluation of pork color by using computer vision," Meat Science, vol. 56, no. 1, pp. 57-60, 2000.

[22] R. Karoui, B. Lefur, C. Grondin et al., "Mid-infrared spectroscopy as a new tool for the evaluation of fish freshness," International Journal of Food Science and Technology, vol. 42, no. 1, pp. 57-64, 2007.

[23] R. Grau, A. J. Sánchez, J. Girón, E. Iborra, A. Fuentes, and J. M. Barat, "Nondestructive assessment of freshness in packaged sliced chicken breasts using SW-NIR spectroscopy," Food Research International, vol. 44, no. 1, pp. 331-337, 2011.

[24] Z. Xiong, D. W. Sun, H. Pu, A. Xie, Z. Han, and M. Luo, "Nondestructive prediction of thiobarbituricacid reactive substances (TBARS) value for freshness evaluation of chicken meat using hyperspectral imaging," Food Chemistry, vol. 179, pp. 175$181,2015$.

[25] G. Elmasry, D. F. Barbin, D. W. Sun, and P. Allen, "Meat quality evaluation by hyperspectral imaging technique: an overview," Critical Reviews in Food Science and Nutrition, vol. 52, no. 8, pp. 689-711, 2012.

[26] H. Li, Q. Chen, J. Zhao, and Q. Ouyang, "Non-destructive evaluation of pork freshness using a portable electronic nose (E-nose) based on a colorimetric sensor array," Analytical Methods, vol. 6, no. 16, pp. 6271-6277, 2014.

[27] L. Huang, J. Zhao, Q. Chen, and Y. Zhang, "Nondestructive measurement of total volatile basic nitrogen (TVB-N) in pork meat by integrating near infrared spectroscopy, computer vision and electronic nose techniques," Food Chemistry, vol. 145, pp. 228-236, 2014.

[28] F. Han, X. Huang, E. Teye, F. Gu, and H. Gu, "Nondestructive detection of fish freshness during its preservation by combin- ing electronic nose and electronic tongue techniques in conjunction with chemometric analysis," Analytical Methods, vol. 6, no. 2, pp. 529-536, 2014.

[29] E. Borràs, J. Ferré, R. Boqué, M. Mestres, L. Aceña, and O. Busto, "Data fusion methodologies for food and beverage authentication and quality assessment - a review," Analytica Chimica Acta, vol. 891, pp. 1-14, 2015.

[30] M. Ghasemi-Varnamkhasti, S. S. Mohtasebi, M. L. Rodriguez-Mendez et al., "Classification of non-alcoholic beer based on aftertaste sensory evaluation by chemometric tools," Expert Systems with Applications, vol. 39, no. 4, pp. 4315-4327, 2012.

[31] M. Śliwińska, P. Wiśniewska, T. Dymerski, J. Namieśnik, and W. Wardencki, "Food analysis using artificial senses," Journal of Agricultural and Food Chemistry, vol. 62, no. 7, pp. 14231448, 2014.

[32] S. Buratti, C. Malegori, S. Benedetti, P. Oliveri, and G. Giovanelli, "E-nose, e-tongue and e-eye for edible olive oil characterization and shelf life assessment: a powerful data fusion approach," Talanta, vol. 182, pp. 131-141, 2018.

[33] L. Lu, S. Deng, Z. Zhu, and S. Tian, "Classification of rice by combining electronic tongue and nose," Food Analytical Methods, vol. 8, no. 8, pp. 1893-1902, 2014.

[34] Q. Chen, J. Zhao, Z. Chen, H. Lin, and D. A. Zhao, “Discrimination of green tea quality using the electronic nose technique and the human panel test, comparison of linear and nonlinear classification tools," Sensors and Actuators B: Chemical, vol. 159, no. 1, pp. 294-300, 2011.

[35] J. Cai, Q. Chen, X. Wan, and J. Zhao, "Determination of total volatile basic nitrogen (TVB-N) content and Warner-Bratzler shear force (WBSF) in pork using Fourier transform near infrared (FT-NIR) spectroscopy," Food Chemistry, vol. 126, no. 3, pp. 1354-1360, 2011.

[36] Ministry of Health of the People's Republic of China, National Standards of the People's Republic of China GB/T 5009.44-2003 (11 August 2003) on the method for analysis of hygienic standard of meat and meat products, China, 2003.

[37] X. Guan, J. Liu, Q. Huang, and J. Li, “Assessing the freshness of meat by using quantum-behaved particle swarm optimization and support vector machine," Journal of Food Protection, vol. 76, no. 11, pp. 1916-1922, 2013.

[38] A. R. Di Rosa, F. Leone, F. Cheli, and V. Chiofalo, "Fusion of electronic nose, electronic tongue and computer vision for animal source food authentication and quality assessment - a review," Journal of Food Engineering, vol. 210, pp. 62-75, 2017.

[39] H. Li, Q. Chen, J. Zhao, and M. Wu, "Nondestructive detection of total volatile basic nitrogen (TVB-N) content in pork meat by integrating hyperspectral imaging and colorimetric sensor combined with a nonlinear data fusion," LWT - Food Science and Technology, vol. 63, no. 1, pp. 268-274, 2015.

[40] K. Timsorn, T. Thoopboochagorn, N. Lertwattanasakul, and C. Wongchoosuk, "Evaluation of bacterial population on chicken meats using a briefcase electronic nose," Biosystems Engineering, vol. 151, no. 1, pp. 116-125, 2016.

[41] J. Chen, J. Gu, R. Zhang, Y. Mao, and S. Tian, "Freshness evaluation of three kinds of meats based on the electronic nose," Sensors, vol. 19, no. 3, p. 605, 2019.

[42] A. Girolami, F. Napolitano, D. Faraone, and A. Braghieri, "Measurement of meat color using a computer vision system," Meat Science, vol. 93, no. 1, pp. 111-118, 2013. 
[43] X. Sun, J. Young, J. H. Liu et al., "Prediction of pork color attributes using computer vision system," Meat Science, vol. 113, no. 62-64, pp. 62-64, 2016.

[44] Y. Li, X. Tang, Z. Shen, and J. Dong, "Prediction of total volatile basic nitrogen (TVB-N) content of chilled beef for freshness evaluation by using viscoelasticity based on airflow and laser technique," Food Chemistry, vol. 287, pp. 126-132, 2019. 